Pacific Journal of Mathematics

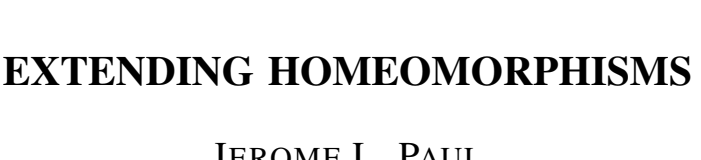




\title{
EXTENDING HOMEOMORPHISMS
}

\author{
Jerome L. Paul
}

Theorem 1 of this paper establishes a necessary and sufficient condition that a locally flat imbedding $f: B^{k} \rightarrow R^{n}$ of a $k$-cell in euclidean $n$-space $R^{n}$ admits an extension to a homeomorphism $F: R^{n} \rightarrow R^{n}$ onto $R^{n}$ such that $F \mid\left(R^{n}-B^{k}\right)$ is a diffeomorphism which is the identity outside some compact set in $R^{n}$. An analogous result for locally flat imbeddings of a euclidean $(n-1)$-sphere into $R^{n}$ is proved. A lemma which generalizes a theorem of Huebsch and Morse concerning Schoenflies extensions without interior differential singularities is also established.

Let the points of euclidean $n$-space $R^{n}$ be written $x=\left(x^{1}, \cdots, x^{n}\right)$, and provide $R^{n}$ with the usual euclidean norm $\|x\|=\left[\Sigma\left(x^{i}\right)^{2}\right]^{1 / 2}$. We set $S_{r}=\left\{x \in R^{n}\|\| x \|=r\right\}$, (and $S=S_{1}$ ). If $M$ is a topological $(n-1)$ sphere in $R^{n}$, we denote the bounded component of $R^{n}-M$ by $\dot{J} M$, and the closure of $\dot{J} M$ in $R^{n}$ by $J M$. We refer the reader to $\S 1$ of [2] for the definition of the terms admissible cone $K_{z}$, conical point, axis of singular approach, and cone $K_{z}(\Sigma)$, where $\Sigma$ is a euclidean $(n-1)$-sphere in $R^{n}$.

Lemma 1. Let $z$ be an arbitrary point of $S$ and $\varphi$ a sensepreserving homeomorphism into $R^{n}$ of an open neighborhood $N$ of $S$ such that $\varphi$ carries points inside $S$ to points inside $\varphi(S)$, and $\varphi \mid(N-S)$ is a $C^{m}$-diffeomorphism. There then exists a homeomorphism $\Phi$ of $R^{n}$ onto $R^{n}$ and a cone $K_{z}$ (resp. $\check{K}_{z}$ ) with axis interiorly normal (resp. exteriorly normal) to $S$ at $z$, such that if $X \subset N$ is a sufficiently small open neighborhood of $S$,

$$
\Phi(x)=\varphi(x) \quad\left[x \in X-\left\{K_{z}(S) \cup \check{K}_{z}\right\}\right],
$$

$\Phi \mid\left(R^{n}-S\right)$ is a $C^{m}$-diffeomorphism, and $\Phi$ is the identity outside some compact set in $R^{n}$.

REMARK. We note that a direct application of the proof of Theorem 1.2 of [2] will yield the conclusions of Lemma 1 except for single differential singularities in each component of $R^{n}-S$.

Proof of Lemma 1. The proof of Lemma 1 will be a variation of the proof of Theorem 1.2 of [2]. We can assume that $0 \in \dot{J}_{\varphi}(S)$. Let $\delta \in\left(\frac{1}{2}, 1\right)$ be a constant so near 1 that $S_{\delta} \subset N$. Using Theorem 1.1 of [2], there is a homeomorphism $f: J S \rightarrow R^{n}$ into $R^{n}$ such that 


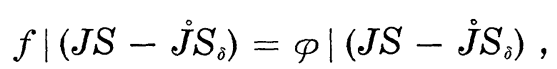

and $f \mid(\dot{J} S-0)$ is a $C^{m}$-diffeomorphism. We can also assume that $f(0)=0$. We now apply Lemma 5.3 of [2] to $f \mid(\stackrel{J}{S} \cap \cap N), S_{\delta}$, and the fixed point $y=\delta z \in S_{\delta}$, and conclude that if $\rho>1$ is a sufficiently large constant, there exists a homeomorphism $\Theta$ of $R^{n}$ onto $R^{n}$ such that $\Theta(x)=f(x)$ for $x \in Y \cup J S_{\tilde{\delta}}$, where $Y$ is a suitable neighborhood of $S_{\delta}, \Theta \mid\left(R^{n}-\{0 \cup \rho z\}\right)$ is a $C^{m}$-diffeomorphism, where the point $\rho z$ is a conical point of $\Theta$ with cone $K_{\rho z}$ of singular approach to $\rho z$ whose axis is interiorly normal to $S_{\rho}$ at $\rho z$, and if a constant $\nu \in(0,1)$ is sufficiently near $1, \Theta$ reduces to the identity on $R^{n}-\left\{B_{\nu \rho} \cup K_{\rho_{z}}\left(S_{\rho}\right)\right\}$.

Let $\omega$ be a radial $C^{\infty}$-diffeomorphism of $R^{n}$ onto $R^{n}$ such that $\omega(x)=$ $x$ for $\|x\| \leqq \varepsilon$, and $\omega(x)=\rho x$ for $\|x\| \geqq 1-\varepsilon$, where $0<\varepsilon<\frac{1}{2}$. We then set $\Phi(x)=f \omega^{-1} \Theta^{-1} \omega(x)$ for $x \in J S$. If $\zeta: R^{n} \rightarrow R^{n}$ is the $C^{\infty}$-diffeomorphism $\zeta(x)=\rho x$, we set $K_{z}=\zeta^{-1}\left(K_{\rho_{z}}\right)$ and $\hat{X}=\zeta^{-1}\left(J S_{\rho}-B_{\nu \rho}\right)$. We can assume that $\nu$ is so near 1 that $1-\varepsilon<\nu$ and $\delta<\nu$, so that $\zeta^{-1}\left(J S_{\rho}-B_{\nu \rho}\right)=\omega^{-1}\left(J S_{\rho}-B_{\nu \rho}\right)$ and $\hat{X} \subset J S-J S_{\hat{o}}$. Then we see that $\Phi\left|\left(\hat{X}-K_{z}(S)\right)=\varphi\right|\left(\hat{X}-K_{z}(S)\right)$ and $\Phi \mid \dot{J} S$ is a $C^{m}$-diffeomorphism (which reduces to the identity on a neighborhood of 0 ). We have therefore defined $\Phi \mid J S$ with the desired properties. We then define $\Phi$ on $R^{n}-\grave{J} S$ in an analogous manner to satisfy the conclusions of Lemma 1 by regarding $R^{n}$, with the "point at infinity" added, as an $n$-sphere, and using the geometry of inversion. This completes the proof of Lemma 1.

REMARK. As the proof of Lemma 1 shows, we also could state corresponding "one-sided" lemmas in which the differentability is only assumed either outside or inside of $S$. For example, if only $\varphi \mid(N-J S)$ is a $C^{m}$-diffeomorphism, then $\check{K}_{z}$ and $\Phi$ exist where $\Phi \mid\left(R^{n}-J S\right)$ is a $C^{m}$-diffeomorphism which is the identity outside some compact set in $R^{n}$.

We now fix the integer $n$, and for any integer $k \leqq n$, we regard $R^{k} \subset R^{n}$ as consisting of those points $x=\left(x^{1}, \cdots, x^{n}\right)$ with $x^{k+1}=\cdots=$ $x^{n}=0$. We denote the unit $k$-cell in $R^{k} \subset R^{n}$ by $B^{k}$. For convenience, we assume in what follows that "diffeomorphism" means " $C^{\infty}$-diffeomorphism."

THEOREM 1. Let $f: B^{k} \rightarrow R^{n}$ be a homeomorphism into $R^{n}$ such that each point $x \in B^{k}$ has an open neighborhood $V_{x}$ in $R^{n}$ and $a$ sense-preserving homeomorphism $f_{x}: V_{x} \rightarrow R^{n}$ into $R^{n}$ satisfying

$$
f_{x}\left|\left(V_{x} \cap B^{k}\right)=f\right|\left(V_{x} \cap B^{k}\right)
$$

and $f_{x} \mid\left(V_{x}-B^{k}\right)$ is a diffeomorphism. Then there exists a homeomorphism $F$ of $R^{n}$ onto $R^{n}$ such that 
(i) $F \mid B^{k}=f$,

(ii) $F \mid\left(R^{n}-B^{k}\right)$ is a diffeomorphism,

(iii) $F$ is the identity outside some compact set in $R^{n}$.

Proof. An examination and easy modification of the proof of Proposition $C$ in [1] shows that there exists an open neighborhood $N$ of $B^{k}$ in $R^{n}$ and a sense-preserving homeomorphism $\varphi: N \rightarrow R^{n}$ into $R^{n}$ such that $\varphi \mid B^{k}=f$ and $\varphi \mid\left(N-B^{k}\right)$ is a diffeomorphism. Let $J \mathscr{S} \subset N$ be a smooth convex $n$-cell in $R^{n}$, where $\mathscr{S}$ is a smooth $(n-1)$-sphere in $R^{n}$ such that $B^{k} \subset \mathscr{S}$, and let $z$ be an arbitrary point in $B^{k}$. Using the remark following Lemma 1, there exists an open neighborhood $Y$ of $\mathscr{S}$ in $R^{n}$, a cone $K_{z}$ with axis exteriorly normal to $\mathscr{S}$ at $z$, and a homeomorphism $\Phi$ of $R^{n}$ onto itself such that $\Phi(x)=\varphi(x)$ for $x \in\left(Y-K_{z}\right)$, and $\Phi \mid\left(R^{n}-J \mathscr{S}\right)$ is a diffeomorphism which is the identity outside some compact subset of $R^{n}$. We then define $F: R^{n} \rightarrow R^{n}$ by

$$
\begin{array}{ll}
F(x)=\varphi(x) & {\left[x \in\left(Y-K_{z}\right) \cup J \mathscr{S}\right]} \\
F(x)=\Phi(x) & {\left[x \in Y \cup\left(R^{n}-J \mathscr{S}\right)\right] .}
\end{array}
$$

It is clear that $F$ satisfies the conclusions of Theorem 1.

Lemma 2. Let $f: R^{n-1} \rightarrow R^{n}, n \geqq 4$ be an imbedding of $R^{n-1}$ as a closed subset of $R^{n}$. Suppose for each $x \in R^{n-1}$ there is a neighborhood $V_{x}$ of $x$ in $R^{n}$ and a homeomorphism $f_{x}: V_{x} \rightarrow R^{n}$ into $R^{n}$ such that $f_{x}\left|\left(V_{x} \cap R^{n-1}\right)=f\right|\left(V_{x} \cap R^{n-1}\right)$, and $f_{x} \mid\left(V_{x}-R^{n-1}\right)$ is a diffeomorphism. Then there is a homeomorphism $F$ of $R^{n}$ onto $R^{n}$ such that $F \mid R^{n-1}=$ $f$ and $F \mid\left(R^{n}-R^{n-1}\right)$ is a diffeomorphism.

Proof. As in the proof of Lemma 2, (cf. Proposition $C_{1}$ of [1]), there is an open neighborhood $U$ of $R^{n-1}$ in $R^{n}$ and a homeomorphism $\Phi: U \rightarrow R^{n}$ into $R^{n}$ such that $\Phi \mid R^{n-1}=f$ and $\Phi \mid\left(U-R^{n-1}\right)$ is a diffeomorphism. Let $\mathscr{R}_{1}^{n-1}, \mathscr{R}_{2}^{n-1}$ be diffeomorphs (under good $C^{1}$ approximations to the inclusion) of $R^{n-1}$ as closed subsets of $R^{n}$ such that $\mathscr{R}_{1}{ }^{n-1}$ and $\mathscr{R}_{2}{ }^{n-1}$ are contained in opposite components of $R^{n}-R^{n-1}$, and if $V$ denotes the component of $R^{n}-\left\{\mathscr{R}_{1}{ }^{n-1} \cup \mathscr{R}_{2}{ }^{n-1}\right\}$ which contains $R^{n-1}$, then $\bar{V}=V \cup \mathscr{R}_{1}{ }^{n-1} \cup \mathscr{R}_{2}{ }^{n-1} \subset U$. Let $V_{1}$ (resp. $V_{2}$ ) denote that component of $R^{n}-\mathscr{R}_{1}{ }^{n-1}$ (resp. $R^{n}-\mathscr{R}_{2}{ }^{n-1}$ ) which does not contain $\mathscr{R}_{2}^{n-1}$ (resp. $\mathscr{R}_{1}^{n-1}$ ). Applying the corollary to Theorem 1 of [3], there are diffeomorphisms $\Theta_{1}, \Theta_{2}$ of $R^{n}$ onto $R^{n}$ such that $\Theta_{i}\left|\mathscr{R}_{i}{ }^{n-1}=\Phi\right| \mathscr{R}_{i}{ }^{n-1}, i=1,2$. Since any orientation-preserving diffeomorphism of $R^{n-1}$ on itself is diffeotopic to the identity, we may assume that $\Theta\left|0_{i}=\Phi\right| 0_{i}$, where $0_{i}$ is an open neighborhood of $\mathscr{R}_{i}^{n-1}$ in $R^{n}-R^{n-1}, i=1,2$. Then $F: R^{n} \rightarrow R^{n}$ defined by 


$$
\begin{array}{ll}
F(x)=\Theta_{1}(x) & {\left[x \in 0_{1} \cup V_{1}\right],} \\
F(x)=\Theta_{2}(x) & {\left[x \in 0_{2} \cup V_{2}\right],} \\
F(x)=\Phi(x) & {\left[x \in 0_{1} \cup 0_{2} \cup V\right]}
\end{array}
$$

satisfies the conclusions of Lemma 2.

Using one point compactification and stereographic projection, Theorem 2 below is obtained readily from Lemmas 2 and 1.

THEOREM 2. Let $f: S \rightarrow R^{n}$ be a homeomorphism into $R^{n}, n \geqq 4$, and let $p$ be an arbitrary point in $S$. Suppose each point $x \in S-p$ has an open neighborhood $V_{x}$ in $R^{n}$ and a sensepreserving homeomorphism $f_{x}: V_{x} \rightarrow R^{n}$ into $R^{n}$ such that $f_{x}\left|\left(V_{x} \cap S\right)=f\right|\left(V_{x} \cap S\right)$, $f \mid\left(V_{x}-S\right)$ is a diffeomorphism, and $f_{x}$ carries points inside $S$ to points inside $f(S)$. Then there is a homeomorphism $F$ of $R^{n}$ onto itself such that $F \mid S=f$, and $F \mid\left(R^{n}-S\right)$ is a diffeomorphism which is the identity outside some compact subset in $R^{n}$.

\section{REFERENCES}

1. A. V. Chernavskii, Homeomorphisms of euclidean spaces and topological imbeddings of polyhedra in euclidean spaces II, Math. USSR Sbornik 1 (1967), 519-541.

2. W. Huebsch and M. Morse, Schoenflies extensions without interior differential singularities, Ann. of Math. 76 (1962), 18-54.

3. J. Stallings, "On infinite processes leading to differentiability in the complement of a point," Differential and combinatorial topology, Princeton Univ. Press, Princeton, New Jersey, 1965.

Received October 8, 1968. The author was partially supported by the National Science Foundation.

PURdue UNIVERSity

LAFAYETTE, INDIANA 


\section{PACIFIC JOURNAL OF MATHEMATICS}

\section{EDITORS}

\author{
H. SAMELSON \\ Stanford University \\ Stanford, California 94305 \\ RICHARD PIERCE \\ University of Washington \\ Seattle, Washington 98105
}

J. DugundJI

Department of Mathematics University of Southern California Los Angeles, California 90007

BASIL GORDON*

University of California

Los Angeles, California 90024

\section{ASSOCIATE EDITORS}
E. F. BECKENBACH
B. H. NEUMANN
F. WOLE
K. YOSHIDA

\section{SUPPORTING INSTITUTIONS}

\author{
UNIVERSITY OF BRITISH COLUMBIA \\ CALIFORNIA INSTITUTE OF TECHNOLOGY \\ UNIVERSITY OF CALIFORNIA \\ MONTANA STATE UNIVERSITY \\ UNIVERSITY OF NEVADA \\ NEW MEXICO STATE UNIVERSITY \\ OREGON STATE UNIVERSITY \\ UNIVERSITY OF OREGON \\ OSAKA UNIVERSITY \\ UNIVERSITY OF SOUTHERN CALIFORNIA
}

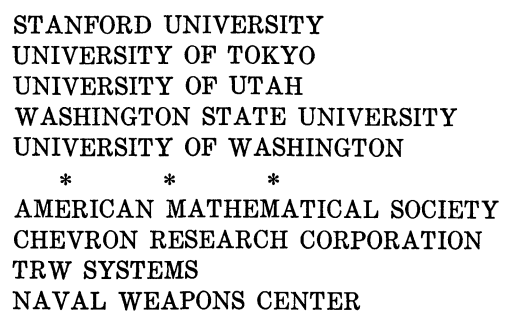

The Supporting Institutions listed above contribute to the cost of publication of this Journal, but they are not owners or publishers and have no responsibility for its content or policies.

Mathematical papers intended for publication in the Pacific Journal of Mathematics should be in typed form or offset-reproduced, (not dittoed), double spaced with large margins. Underline Greek letters in red, German in green, and script in blue. The first paragraph or two must be capable of being used separately as a synopsis of the entire paper. The editorial "we" must not be used in the synopsis, and items of the bibliography should not be cited there unless absolutely necessary, in which case they must be identified by author and Journal, rather than by item number. Manuscripts, in duplicate if possible, may be sent to any one of the four editors. Please classify according to the scheme of Math. Rev. 36, 1539-1546. All other communications to the editors should be addressed to the managing editor, Richard Arens, University of California, Los Angeles, California, 90024.

50 reprints are provided free for each article; additional copies may be obtained at cost in multiples of 50 .

The Pacific Journal of Mathematics is published monthly. Effective with Volume 16 the price per volume (3 numbers) is $\$ 8.00$; single issues, $\$ 3.00$. Special price for current issues to individual faculty members of supporting institutions and to individual members of the American Mathematical Society: $\$ 4.00$ per volume; single issues $\$ 1.50$. Back numbers are available.

Subscriptions, orders for back numbers, and changes of address should be sent to Pacific Journal of Mathematics, 103 Highland Boulevard, Berkeley, California, 94708.

PUBLISHED BY PACIFIC JOURNAL OF MATHEMATICS, A NON-PROFIT CORPORATION

Printed at Kokusai Bunken Insatsusha (International Academic Printing Co., Ltd.), 7-17, Fujimi 2-chome, Chiyoda-ku, Tokyo, Japan.

* Acting Managing Editor. 


\section{Pacific Journal of Mathematics}

\section{Vol. 32, No. $2 \quad$ February, 1970}

Harry P. Allen and Joseph Cooley Ferrar, Jordan algebras and exceptional subalgebras of the exceptional algebra $E_{6} \ldots \ldots \ldots \ldots \ldots \ldots \ldots 283$

David Wilmot Barnette and Branko Grünbaum, Preassigning the shape of a

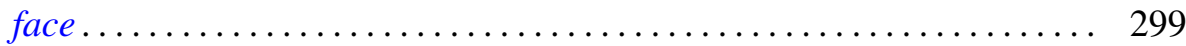

Robert Francis Craggs, Involutions of the 3-sphere which fix 2-spheres . . . . 307

David William Dean, Bor-Luh Lin and Ivan Singer, On k-shrinking and $k$-boundedly complete bases in Banach spaces ................ 323

Martin Engert, Finite dimensional translation invariant subspaces ....... 333

Kenneth Lewis Fields, On the global dimension of residue rings ......... 345

Howard Gorman, The Brandt condition and invertibility of modules ....... 351

Benjamin Rigler Halpern, A characterization of the circle and interval ..... 373

Albert Emerson Hurd, A uniqueness theorem for second order quasilinear

hyperbolic equations ............................... 415

James Frederick Hurley, Composition series in Chevalley algebras ...... 429

Meira Lavie, Disconjugacy of linear differential equations in the complex

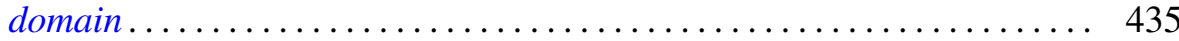

Jimmie Don Lawson, Lattices with no interval homomorphisms ......... 459

Roger McCann, A classification of center-foci ................. 467

Evelyn Rupard McMillan, On continuity conditions for functions . . . . . . . 479

Graciano de Oliveira, A conjecture and some problems on permanents .... 495

David L. Parrott and S. K. Wong, On the Higman-Sims simple group of order $44,352,000$.

Jerome L. Paul, Extending homeomorphisms ................. 517

Thomas Benny Rushing, Unknotting unions of cells .............. 521

Peter Russell, Forms of the affine line and its additive group.......... 527

Niel Shilkret, Non-Archimedean Gelfand theory ................. 541

Alfred Esperanza Tong, Diagonal submatrices of matrix maps.......... 551 